\title{
Antidopaje en el deporte: el impacto de los programas educativos antidopaje para las federaciones deportivas
}

\section{Anti-doping in sport: the impact of anti-doping educational programs for sports federations}

\author{
DOI: $10.46932 / s f j d v 3 n 2-004$
}

Received in: February $28^{\text {th }}, 2022$

Accepted in: March $1^{\text {st }}, 2022$

\author{
Túlia Marques Martinó \\ Institución: University Évora
}

Dirección: Estrada das Galegas Edifício Marvâo Norte Cave A 2640-049 Santo Isidoro- Mafra-Portugal

Correo electrónico: tulocas@ gmail.com

\author{
Maria Céu Machado \\ Degree in Mediciner \\ Institución: University Évora
}

Dirección: Estrada das Galegas Edifício Marvâo Norte Cave A 2640-049 Santo Isidoro- Mafra-Portugal Correo electrónico: ceu.machado.pt@gmail.com

\section{Mário Rui Coelho Teixeira}

Ph.D. Sports Management

Institución: University Évora

Dirección: Estrada das Galegas Edifício Marvâo Norte Cave A 2640-049 Santo Isidoro- Mafra-Portugal Correo electrónico: mario.teixeira.pt@gmail.com

\section{RESUMEN}

Meta: Analizar el impacto que las acciones educativas antidopaje tienen dentro de las federaciones deportivas, en Portugal y España. ¿Cómo están a ser implementados dentro de las federaciones deportivas, en los dos países, los programas educativos contra el dopaje? ¿Cómo se desarrollan y se divulgan? ¿Cuál es su éxito? Metodología: de la muestra forman parte 30 federaciones deportivas nacionales, 15 portuguesas y 15 españolas. El criterio de elección se basó en las federaciones deportivas portuguesas con deportistas federados en el año 2014. Para las dimensiones del estudio, además del número de formaciones, tuvimos en cuenta el número de deportistas federados cuyo resultado en dopaje fue positivo. El correo electrónico fue el procedimiento utilizado para solicitar a todas las federaciones el número total de atletas federados y los resultados positivos en dopaje, así como el número de acciones de formación antidopaje. Se utilizó como método el análisis de los porcentajes de resultados positivos de dopaje en función del número de formaciones, tal como los resultados positivos en función del año y de la comparación entre las federaciones. Resultados: (análisis 1), los datos demuestran que existe una relación positiva entre el número de formaciones antidopaje y la disminución de casos positivos. (análisis 2), hay tendencia a existir un mayor número de casos positivos en años pares, (análisis 3), se concluye que los deportes realzados tienen comportamiento similar. Conclusiones: cuanto mayor es el número de acciones de formación antidopaje, menor es el número de resultados positivos de dopaje.

Palabras clave: gestión del deporte, ética, medicina, antidopaje, federaciones deportivas. 


\begin{abstract}
Objective: To analyse the impact that anti-doping educational actions have within the sports federations, in Portugal and Spain. How are anti-doping educational programs to be implemented within sports federations in both countries? How are they developed and disseminated? What is your success? Methodology: 30 national sports federations, 15 Portuguese and 15 Spanish federations form part of the sample. The election criteria was based on the Portuguese sports federations with federated athletes in 2014. For the dimensions of the study, in addition to the number of training, we took into account the number of federated athletes whose results in doping was positive. The e-mail was the procedure used to request all federations the total number of federated athletes and the positive results in doping, as well as the number of anti-doping training actions. The method used was the analysis of the percentages of positive results of doping according to the number of training sessions, such as the positive results depending on the year and the comparison between the federations. Results: (analysis 1), the data show that there is a positive relationship between the number of anti-doping training and the reduction of positive cases. (analysis 2), there is a tendency to have a greater number of positive cases in even years, (analysis 3), it is concluded that enhanced sports have a similar behaviour. Conclusions: the greater the number of anti-doping training actions, the lower the number of positive doping results
\end{abstract}

Keywords: sports management, ethic, medicine, antidoping, sports federations.

\title{
1 INTRODUCCIÓN
}

El ideal deportivo en su génesis es precisamente la igualdad de oportunidades, defiende que gane lo mejor, lo que tiene mayor capacidad y que más ha trabajado para alcanzar la victoria. Todos estos ideales, hacen del espacio deportivo una escuela de virtudes, de preparación para la vida, de formación de una moral de respeto por la dignidad de unos con otros (Marivoet, 1998).

La utilización de sustancias dopantes se remonta desde el principio de los tiempos. En la antigua Grecia, alrededor del año 800 A.C. los griegos incorporaron el deporte en su estilo de vida, como una costumbre cultural y religiosa (López, 2010). La dieta del atleta, consistía en hongos, alucinógenos, semillas de plantas, higos secos, vino, queso o carne húmeda (Baron, Martin, \& Abol, 2007, Higgins, 2006). Los romanos tenían como entretenimiento, luchas entre gladiadores y las carreras de coches (Rodríguez, 2008). Los romanos alimentaban a los gladiadores, con mezcla de estimulantes y alcohol, a fin de evitar la fatiga y las luchas ser de mayor violencia (Ramos, 1999). No século XVI, se inicia el uso de drogas para fines militares. La cocaína, la heroína, la anfetamina o la morfina se utilizaron durante la Guerra Civil de los Estados Unidos, la guerra de Alemania en 1883 (Bahrke \& Yesalis, 2002; Mataix, Sanchez, \& González, 2006).

En la segunda gran guerra, se inicia una nueva era de sustancias farmacológicas en la esfera deportiva y militar, por ejemplo, efedrina, testosterona y anfetaminas (López, 2010).

Las primeras competiciones de ciclistas, ocurren en el año 1879, es por esta época que la primera muerte por dopaje ocurre, con el ciclista inglés, Arthur Linton, muere durante la competición de 
Bordeaux-París, en el año 1886 (Barbany, 2002). La administración de narcóticos a los caballos de carreras, práctica común en la edad media, se mantiene hasta 1912, cuando se realizan los primeros análisis de la saliva de los animales (Mazanov \& McDermott, 2009). En la década del 60, surge el "doping hormonal", que se hizo conocido debido al consumo de esteroides anabolizantes, por parte de los atletas americanos, con el propósito de aumentar la masa muscular, (Mataix et al., 2006).

La lucha contra el dopaje cuenta con la colaboración de gobiernos, organizaciones y entidades deportivas. La Convención Internacional, establece en el artículo 19 la creación e implementación de programas de educación y formación sobre el Doping, y deberán figurar en estos información actualizada y precisa sobre los daños de Doping a los valores éticos del deporte; las consecuencias para la salud, los derechos y las responsabilidades de los atletas, información sobre el Código y las políticas antidopaje, la lista de sustancias y métodos prohibidos, las autorizaciones de uso terapéutico y la información sobre los complementos nutricionales .

El principio básico para los programas de información y educación es preservar el espíritu deportivo según lo descrito en el Código, así como proveer a los atletas con información, valores y principios para que éstos lleven una vida deportiva limpia y libre del Dopaje. ¿Están las organizaciones deportivas a prestar atención adecuada a esta temática?

Este estudio tiene como objetivo comprender el impacto de los programas educativos antidopaje de las Federaciones deportivas españolas y portuguesa. ¿Cuál es su éxito? Se procedió al análisis y comparación de los datos estadísticos referentes al Doping entre las modalidades individuales y colectivas, para responder a esta cuestión.

\section{MÉTODO}

Muestra seleccionada para este estudio se basa en 15 Federaciónes Portuguesas de Deportes y 15 federaciones españolas de deportes individuales y colectivos, siendo estos: Real Athletic Federación, Balonmano, ciclismo, automovilismo, tiro olímpico, golf, karate , Natación, Tenis, Taekwondo, Tiro Al Vuelo, Voleibol, Real Federación Hípica Baloncesto y Rugby.

Instrumento: entrevistas por teléfono, análisis documental, análisis de contenido.

Criterios de selección: las federaciones deportivas portugués con los atletas más registrados en 2014, teniendo en cuenta todos los niveles de competencia.

Hipótesis de estudio: número de acciones formativas su impacto en el seno de las Federaciones de ambos países, evaluar a través de los casos positivos de dopaje cada año, cada federación, recogida, tratamiento y análisis de los datos. El modelo de análisis: número de atletas inscritos, el número de casos positivos y de acciones formativas entre 2004 a 2014. 
Las dimensiones del estudio: número de atletas federados y el número de atletas federados con resultados positivos de dopaje.

Indicadores: número de atletas controlados anualmente en comparación con el número de atletas federados.

Las variables: resultados positivos de dopaje y el número de atletas federados.

Recogida de datos: contacto de las Federaciones Deportivas de ambos países, ADOP, WADA, AEPSAD (Agencia Española de Protección de la salud en el deporte) y la Confederación Española de Deportes. Las técnicas de estadísticas que se utilizarán se basan en tres análisis diferentes: en el porcentaje de resultados positivos de dopaje en función del número de formaciones educativas antidopaje, porcentaje de resultados positivos en función del año, para analizar el componente temporal y su evolución (grandes eventos deportivos como campeonatos del Mundo o Juegos Olímpicos), este análisis se debe a la ausencia de acciones de formación antidopaje en el seno de las respectivas Federaciones o falta de información sobre las mismas por parte de las Federaciones Deportivas, por último se realizó una comparación entre Federaciones Deportivas. Para el primer análisis se hizo correlación de las variables, análisis de regresión lineal, varianza a la regresión y el análisis de máximos y mínimos con la pretensión de analizar la relación entre ellos y relación temporal, es decir, si ocurren en el mismo año o en años consecutiva. Para el segundo análisis se procedió a la evaluación de la regresión lineal y su varianza.

Para el último análisis se compararon las medias, con varianzas desconocidas, se utilizó el test Tstudent para el porcentaje de resultados positivos y para el número de acciones de formación, y se realizó también un análisis de frecuencias. Se espera obtener como resultados una correlación de variables negativa, una pendiente negativa en el estudio de regresión lineal y una baja varianza de regresión, en el primer análisis. En cuanto al segundo análisis se espera un declive negativo en la regresión lineal y resultados bajos en la varianza de regresión.

\section{RESULTADOS}

Se realizaron 3 análisis estadísticos diferentes, para la realización de este trabajo, siendo cada una de ellas presentada a continuación. En este capítulo hemos optado por acercarse a la Federación de Voleibol portugués (FPV) que se destacó en este estudio.

En el primer análisis, la FDV, presenta una correlación entre las variables, un porcentaje de resultados positivos de dopaje y número de formaciones educativas antidopaje negativa (-60.34\%), lo que valida la teoría de que con la existencia de acciones de formación antidopaje, el número de resultados positivos de dopaje disminuye. El valor absoluto de la correlación es sensiblemente cerca del 100\%, lo que indica que en este caso hay una correlación negativa sensiblemente fuerte entre las variables 
analizadas. En el caso de que se produzca un cambio en el valor de la variable, se debe tener en cuenta que, en el caso de que se produzca un error, aplicando la regresión lineal, la recta obtenida presenta una pendiente negativa (-0.00748) y una varianza reducida (0.00712), lo que demuestra una vez más la teoría arriba mencionada.

el segundo análisis realizado, la FPV presenta una correlación entre las variables porcentual de resultados positivos de dopaje y año positivo $(24,86 \%)$, obteniendo un valor-p de 0,461 no rechazando la hipótesis para cualquier nivel de significancia, lo que indica que el porcentaje de resultados positivos de dopaje aumenta con el paso del tiempo (con el aumento de los años), apuntando a un decrecimiento control antidopaje con el paso de los años.

El valor absoluto de la correlación es sensiblemente cercano al 0\%, lo que indica que en este caso hay una correlación positiva sensiblemente débil entre las variables analizadas. En la aplicación de la regresión lineal, la recta obtenida presenta una pendiente positiva $(0,00233)$ y una varianza baja (0,008654), comprobando el análisis anterior (véase gráfico 1, 2 y tabla 1).

Para este análisis se aplicó también la ANOVA (análisis de varianza), obteniendo los siguientes resultados:

\begin{tabular}{|c|c|c|c|c|}
\hline & $g l$ & $S Q$ & $M Q$ & $F$ \\
\hline Regressão & 1 & 0,000597 & 0,000597 & 0,592974 \\
\hline Residual & 9 & 0,009062 & 0,001007 & \\
\hline Total & 10 & 0,009659 & & \\
\hline
\end{tabular}

En el último análisis, al comparar la FPV se constató lo siguiente:

La FPV vs. La Federación deportiva de Rugby, la prueba indica que tanto en\% positivo, como en número de formaciones, la hipótesis nula no debe rechazarse, es decir, no se rechaza que los promedios de los valores sean iguales, por tanto, en\% positivo, en un número de formaciones, ambas tienen comportamientos similares, para un nivel de significancia del 5\%, obteniendo un valor-p de 0,432 y 0,511, respectivamente. En la comparación entre el FPV y el balonmano Federación Portuguesa, Testudiante, nos dice que tanto el análisis de los aspectos positivos\%, y el número de formaciones, la hipótesis nula, no debe ser rechazado, es decir, debe y en el caso de que las medias de los valores sean iguales, tanto en\% positivo, como en número de formaciones, ambas tienen comportamientos similares, para un nivel de significancia del 5\%, obteniendo un valor-p de 0,086 y 0,160.

En lo que se refiere al análisis estadístico de las Federaciones deportivas de España(FDE) y el control de dopaje, se obtuvo poca información debido a la dificultad de obtener colaboración por parte de las mismas. En respuesta a varios intentos de contacto con las FDE, se nos indicó que la información solicitada sería confidencial. Enviándonos directamente a la AEPSAD y al CSD. 
El CSD envió nuestro pedido a la AEPSAD, afirmando que ésta es la poseedora de los datos deseados. La AEPSAD, en respuesta indica que todos los datos se encuentran en los "Anuario de Estadísticas Deportivas" existentes desde el año 2013.

Después de consultar los mismos, detectamos que la información pretendida se encuentraba limitada, o que condicio el estudio . En resumen, al analizar los datos se concluyó: En el año 2011 hasta el año 2014 existe un descenso en la toma de muestras, en el año 2011 se realizaron un total de 5.748 análisis y en 2014 sólo 3843 análisis. La razón por la cual esto sucede es de todo desconocida(ver el número total de muestras gráfico 3 y tabla 2).

Gráfico 1- Análisis estadístico 1 : Porcentaje de resultados positivos de dopaje en función del número de formaciones educativas antidopaje de la FDV.

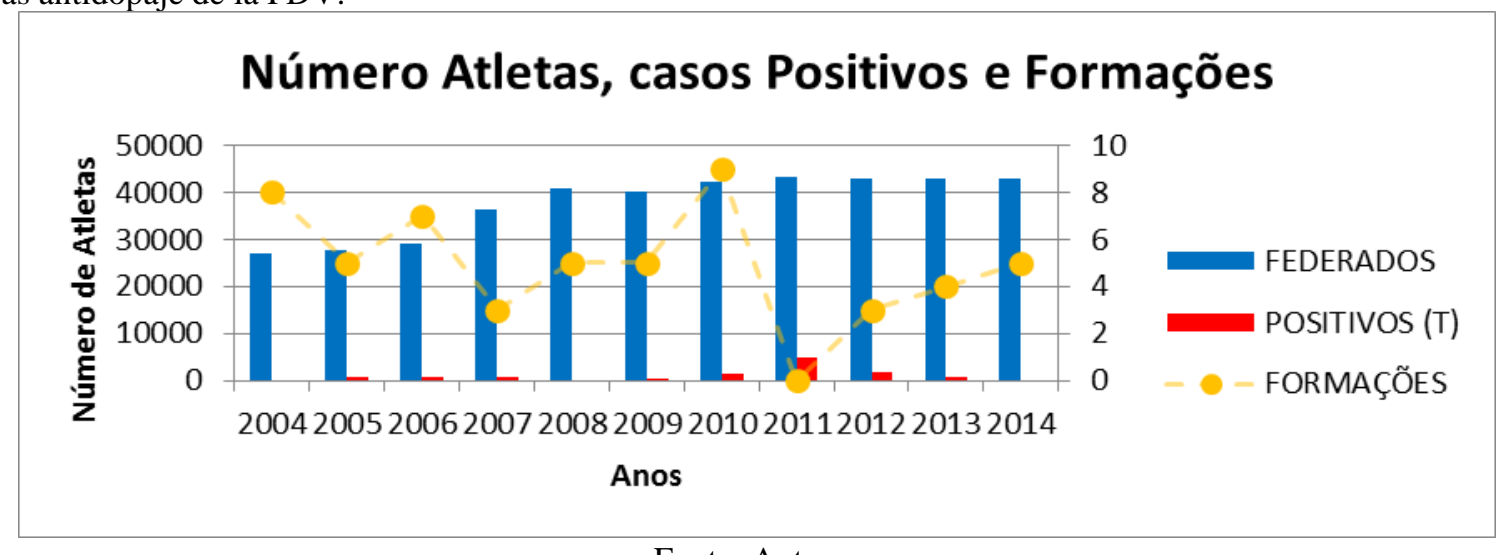

Fonte: Autores

(Gráfico 2- Análisis estadístico 2: Porcentaje de resultados positivos en función del año de la FDV.

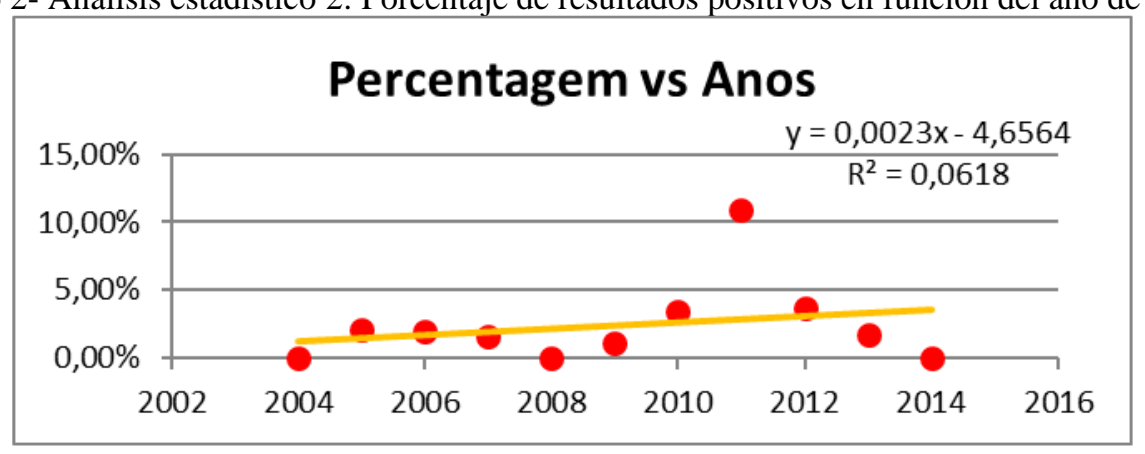

Fonte:Autores

Tabela 2- Total de las Muestra de la FDE e sus resultados.

\begin{tabular}{|c|c|c|c|c|}
\hline ANO & 2011 & 2012 & 2013 & 2014 \\
\hline $\begin{array}{c}\text { TOTAL } \\
\text { MUESTRAS }\end{array}$ & 5.748 & 5402 & 3761 & 3843 \\
\hline $\begin{array}{c}\text { RESULTADO } \\
\text { NEGATIVO }\end{array}$ & 5686 & 5356 & 3732 & 3819 \\
\hline $\begin{array}{c}\text { RESULTADO } \\
\text { ADVERSO }\end{array}$ & 62 & 46 & 29 & 24 \\
\hline
\end{tabular}

Fonte: AEPSAD 
(Gráfico 3- Resultados del control dopagem en Espanha.

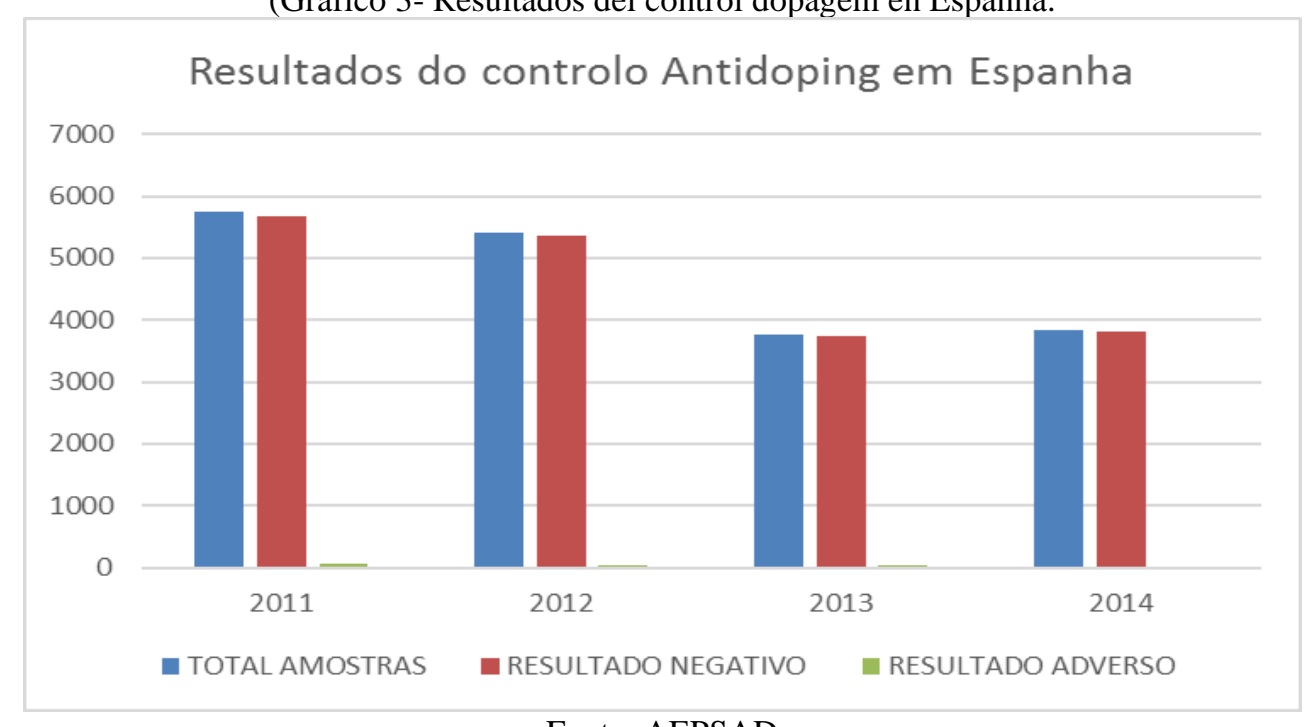

Fonte: AEPSAD

\section{DISCUSIÓN/ CONCLUSIÓN}

Debido a la inexistencia de bases de datos o a no colaboración, sólo 14 Federaciones fueron incluidas en este estudio, de las cuales se destacó la FDV.

La FPV presenta en 2010 el mayor número de acciones de formación, el año 2011 fue el que tuvo mayor número de casos positivos de dopaje, ocho casos positivos de dopaje, cero acciones formativas antidopaje y 73 atletas objetivo de análisis, este fue un año de Precalificación para los Juegos Olímpicos (DO) de Londres en 2012. En 2012, los positivos descendieron drásticamente a cero, en 54 atletas analizados con tres acciones formativas.

En 2014, hubo 5 acciones formativas, cero positivos en 58 atletas analizados.

Utilizando esta federación como ejemplo del estudio se concluye que cuanto mayor sea el número de acciones de formación antidopaje, menor el número de resultados positivos de dopaje. Para obtener la información deseada en Espanã, se estableció contacto con la Agencia Española de Protección de la salud en el Deporte (AEPSAD) y el Consejo Superior de Deportes (CSD) y respectiva Federación de Deportes que corresponden a la Federación de Deportes portuguesas

No se pudo representar estadísticamente a las federaciones deportivas deseadas para este trabajo por falta de datos.

Independientemente de cómo cada país combate el dopaje, los datos demuestran que la implementación de programas educativos antidopaje está teniendo el resultado deseado, la conclusión de los análisis realizados a ambos países dejan claro que las violaciones a las normas antidopaje deben ser extinguidas, independientemente de que éstas se cometan de forma intencionada o ardilosa. Los programas antidopaje informativos y educativos deberían incluir a todas las personas relacionadas con el 
deporte y no a los atletas. Todos deben ser educados sobre el dopaje, las reglas antidopaje, lista de sustancias, los maleficios para la salud y para el espíritu deportivo. (Gibbons, Ebbeck and Weiss, 1995).

(Tabla 1-Visión general de los datos recogidos de la FDV.

\begin{tabular}{|c|c|c|c|c|c|c|}
\hline & FEDERADOS & ANALIZADO & POSITIVOS & FORMACIONES & PORCENTAJE & $\begin{array}{c}\text { POSITIVOS } \\
\text { (T) }\end{array}$ \\
\hline 2004 & 27003 & 54 & 0 & 8 & $0,00 \%$ & 0 \\
\hline 2005 & 27740 & 48 & 1 & 5 & $2,08 \%$ & 578 \\
\hline 2006 & 29135 & 50 & 1 & 7 & $2,00 \%$ & 583 \\
\hline 2007 & 36244 & 64 & 1 & 3 & $1,56 \%$ & 566 \\
\hline 2008 & 40898 & 96 & 0 & 5 & $0,00 \%$ & 0 \\
\hline 2009 & 40090 & 97 & 1 & 5 & $1,03 \%$ & 413 \\
\hline 2010 & 42386 & 59 & 2 & 9 & $3,39 \%$ & 1437 \\
\hline 2011 & 43240 & 73 & 8 & 0 & $10,96 \%$ & 4739 \\
\hline 2012 & 43061 & 54 & 2 & 3 & $3,70 \%$ & 1595 \\
\hline 2013 & 43023 & 60 & 1 & 4 & $1,67 \%$ & 717 \\
\hline 2014 & 43076 & 58 & 0 & 5 & $0,00 \%$ & 0 \\
\hline
\end{tabular}

Fonte : ADOP, autores. 


\section{REFERENCIAS}

Bahrke M., Yesalis C. (2002). Performance-enhancing substances in sport and exercise. Champaign, IL: Human Kinetics.

Barbany, J.R. (2002). Fisiología del ejercicio físico y el entrenamiento. Barcelona: Paidotribo.

Baron, D., Martin, D., \& Abol, S. (2007). El dopaje en el deporte y su propagación a las poblaciones de riesgo: una revisión internacional. World Psychiatry (Edición española), 5(2): 118-123.

Gracia, L., Rey, J.P. \& Casajús, J.A. (2009). El dopaje en los Juegos Olímpicos de verano (19682008). Apunts: Medicina; 44(162), 66-73.

Gibbons, S. L.; Ebbeck, V.; Weiss, M. R. (1995). Fair Play for Kids: Effects on the Moral Development of Children in Physical Education. Quarterly for Exercise and Sport, 66 (3), 247-255. Retrieved from http://www.tandfonline.com/doi/abs/10.1080/02701367.1995.10608839

doi:10.1080/02701367.1995.10608839

Higgins, A.J. (2006). From ancient Greece to modern Athens: 3000 years of doping in competition horses. Journal of Veterinary Pharmacology and Therapeutics, 29(1), 4-8.

doi: 10.1111/j.1365-2885.2006.00770_4.x

Laudo, C., Puigdevall, V., Río, M. J. del, \& Velasco, A. (2006). Hormonas utilizadas como agentes ergogénicos: situación actual del problema. Anales del Sistema Sanitario de Navarra, 29(2), 207-217.

López, S. (2010). Evolución del dopaje en el deporte. Trances, 2(1), 30-54.

Marivoet, S. (1998). Aspetos Sociológicos do Desporto. Lisboa: Livros Horizonte.

Mataix, J., Sanchez, P., \& González, J. (2006). Nutrición en el deporte: ayudas ergogénicas y dopaje. Madrid: Diaz de Santos.

Mazanov, J., McDermott, V. (2009). The case for a social science of drugs in sport. Sport in society, 12(3), 275-295.

Ramos, A.S. (1999). Lucha contra el dopaje como objetivo de salud. Adicciones, 11(4), 299-310. doi: 10.20882/adicciones.609

Rodríguez, C. (2008) La historia del dopaje. Historia del dopaje, sustancias y procedimientos de control. Vol. 1. Madrid: CSD. 